\title{
CMOST: an open-source framework for the microsimulation of colorectal cancer screening strategies
}

Meher K. Prakash', Brian Lang ${ }^{2,3}$, Henriette Heinrich', Piero V. Valli', Peter Bauerfeind', Amnon Sonnenberg ${ }^{4}$, Niko Beerenwinkel ${ }^{2,3}$ and Benjamin Misselwitz ${ }^{1 *}$

\begin{abstract}
Background: Colorectal cancer (CRC) is a leading cause of cancer-related mortality. CRC incidence and mortality can be reduced by several screening strategies, including colonoscopy, but randomized CRC prevention trials face significant obstacles such as the need for large study populations with long follow-up. Therefore, CRC screening strategies will likely be designed and optimized based on computer simulations. Several computational microsimulation tools have been reported for estimating efficiency and cost-effectiveness of CRC prevention. However, none of these tools is publicly available. There is a need for an open source framework to answer practical questions including testing of new screening interventions and adapting findings to local conditions.
\end{abstract}

Methods: We developed and implemented a new microsimulation model, Colon Modeling Open Source Tool (CMOST), for modeling the natural history of CRC, simulating the effects of CRC screening interventions, and calculating the resulting costs. CMOST facilitates automated parameter calibration against epidemiological adenoma prevalence and CRC incidence data.

Results: Predictions of CMOST were highly similar compared to a large endoscopic CRC prevention study as well as predictions of existing microsimulation models. We applied CMOST to calculate the optimal timing of a screening colonoscopy. CRC incidence and mortality are reduced most efficiently by a colonoscopy between the ages of 56 and 59; while discounted life years gained (LYG) is maximal at 49-50 years. With a dwell time of 13 years, the most cost-effective screening is at 59 years, at $\$ 17,211$ discounted USD per LYG. While cost-efficiency varied according to dwell time it did not influence the optimal time point of screening interventions within the tested range.

Conclusions: Predictions of CMOST are highly similar compared to a randomized CRC prevention trial as well as those of other microsimulation tools. This open source tool will enable health-economics analyses in for various countries, health-care scenarios and CRC prevention strategies. CMOST is freely available under the GNU General Public License at https://gitlab.com/misselwb/CMOST

Keywords: Colorectal cancer, Screening colonoscopies, Screening intervals, Cost-effectiveness, Microsimulation

\footnotetext{
* Correspondence: benjamin.misselwitz@usz.ch

'Division of Gastroenterology, University Hospital Zurich (USZ), Rämistrasse

100, 8091 Zurich, Switzerland

Full list of author information is available at the end of the article
} 


\section{Background}

Colorectal cancer (CRC) is the second most common deadly cancer in the United States [1] and many other Western countries. It represents an important public health burden in industrialized countries and increasingly also in developing countries [2]. Since treatment options for advanced cancer are limited, current public health strategies focus on CRC screening for prevention of mortality.

$\mathrm{CRC}$ is remarkable for the long-term presence of adenomatous carcinoma precursors [3, 4]. Early adenomas are, by definition, adenomas with a tubular histology and a size of less than $10 \mathrm{~mm}$. Advanced adenomas are either larger than $10 \mathrm{~mm}$ in size or display an advanced villous or serrated histology. Adenomas are more frequent in males and their frequency increases with age. While adenomas are infrequently observed before the age of 30 years, within the screening population of 5080 year olds, prevalence rates of at least one early adenoma and at least one advanced adenoma are 30\% and $6 \%$, respectively [5]. The presence of adenomas is an important risk factor for subsequent CRC [6].

The natural history of CRC, i.e., the sequence of events leading to CRC in each individual patient, has not been sufficiently clarified. For instance, the adenoma dwell time, i.e., the average time from initiation of an adenoma until initiation of CRC, remains unknown [7, 8]. Furthermore, several lines of evidence indicate that right sided CRC (close to cecum) and left sided CRC (close to rectum) differ regarding their biological behavior: a significant fraction of right-sided CRC derives from serrated adenomas with possible faster progression rates and lower detectability during endoscopy $[9,10]$.

Endoscopy is an attractive method for CRC screening because adenomas can be removed by polypectomy during this intervention, thus effectively preventing CRC [6]. Additional benefits of CRC screening include identification of high-risk individuals for surveillance via the presence of adenomas. Furthermore, screening detects CRC at earlier stages with better survival as compared to symptomatic cancer [11]. The effectiveness of rectosigmoidoscopy, which only visualizes the left-sided colon, in reducing CRC incidence and mortality was demonstrated in several large randomized controlled studies [12-16]. Colonoscopy, which visualizes the whole colon, may be similarly effective as rectosigmoidoscopy [17-20], but rigorous randomized controlled trials have been initiated only recently [21]. As such, the true magnitude of CRC risk reduction by colonoscopy is still unknown. Despite these limitations, colonoscopy screening programs have been implemented in several industrialized countries. It is generally recommended that individuals between ages 50-75 years undergo screening every 10 years, an interval which has not been determined in any systematic way [22]. Alternative CRC screening methods that have also been shown to reduce CRC mortality include detection of fecal occult blood in the stool as a cancer biomarker [23].

The optimal application of CRC screening remains a central task in gastroenterology. However, most of the open questions, such as optimal timing of multiple screening colonoscopies, will likely never be answered by high-quality clinical studies. Randomized trials of CRC screening would need a long follow-up of at least 10 years, its costs are unlikely to be covered by commercial sponsors, and patient compliance might be low due to the (largely unwarranted) perception of colonoscopy being unpleasant or dangerous. Due to these limitations, alternative strategies for studying CRC prevention using computer simulations have been developed.

In microsimulations, a large, simulated, population of many individual patients is followed throughout individual lifetimes for colonic lesions. Microsimulations account for the individual risk for colorectal cancer, the age-dependent gradual appearance of adenomas, the development of adenomas into cancer, and the detection and treatment of CRC. Assumptions about occurrence and growth rates of adenomas are calibrated to reflect the natural history of CRC. Further, the effect of screening interventions and polypectomy can be incorporated into the model. Simulation experiments are appealing, because a calibrated and validated model allows for the assessment of a wide range of medical screening interventions, such as multiple screening colonoscopies, in a time-efficient and cost-effective manner.

Three microsimulation models, namely Microsimulation Screening Analysis (MISCAN), Colorectal Cancer Simulated Population model for Incidence and Natural history (CRC-SPIN), and Simulation Model of Colorectal Cancer (SimCRC) have been described (http://cisnet.cancer.gov/colorectal/ [24]). These models have been very useful to evaluate various screening interventions for CRC with a high degree of confidence [25-29], and they share several features. All models assume that carcinomas develop exclusively from adenomas. Adenomas progress to advanced adenomas or cancer following specific rules and parameters are fit to match the epidemiologically observed prevalence of adenomas, advanced adenomas, and cancer. However, individual model predictions differ and depend on model assumptions and on the choice of parameters [8].

Adenoma dwell time, defined as the time from the appearance of clinically detectable adenoma to the appearance of carcinoma, presents the window of opportunity for effective screening. However, the value of this critical parameter remains unknown. It cannot be determined empirically since adenomas cannot be left in situ. The published microsimulation models assume different values: MISCAN assumes a short adenoma dwell time of 6 years, resulting in rapid adenoma progression and aggressive behavior. By contrast, SimCRC and CRC-SPIN 
use a dwell time of 23 and 18 years, respectively, leading to slow adenoma progression and more benign behavior [30]. Differences of MISCAN, SimCRC, and CRC-SPIN are not restricted to adenoma dwell time, and it remains unknown which model features are responsible for differences in predictions. Further, even though general features of all microsimulation models are described, the models are not publicly available and hence predictions of these models cannot be independently reproduced or advanced.

There is a growing awareness towards CRC screening and its costs and a constant need to determine the costeffectiveness of various screening strategies. Furthermore, many new countries with different CRC epidemiology and health-care costs will be implementing CRC screening programs. Thus, there is an immediate requirement for an open source tool that is transparent, easily accessible, and adaptable for addressing highly relevant clinical and health economy questions.

Here, we present Colon Modeling Open Source Tool (CMOST), a new, open-source CRC microsimulation model. We calibrated our model against clinical data including a large rectosigmoidoscopy screening trial and compared it to existing microsimulation tools. We developed three parameterizations of our model differing in adenoma dwell time. Our results show that adenoma dwell time influences some but not all predictions of our CRC microsimulation model. The model offers the flexibility to design new screening protocols and costs as well as to reparameterize for the natural history by working with the automated calibration.

\section{Methods \\ Characteristics of the CMOST microsimulation model}

We developed a new microsimulation model for CRC, focusing on the natural history as observed by endoscopy on a macroscopic and histological level. The model has been implemented in Matlab as the program Colon Modeling Open Simulation Tool (CMOST). In our model, colorectal cancer may develop either through adenomatous precursors detected by colonoscopy or in a non-adenomatous pathway. Once an adenoma has been transformed into cancer, it can be detected in an asymptomatic state during diagnostic examinations such as endoscopy or when it becomes symptomatic (Fig. 1). The pseudocode illustrating how our CMOST microsimulation program works is given in Table 1.

In our model, an adenoma develops within one of 13 colon segments (Fig. 2). This location remains constant for the lifetime of a lesion affecting both rate of progression as well as accessibility and detection by endoscopic methods such as rectosigmoidoscopy and colonoscopy. Altogether 6 distinct adenoma stages are distinguished: Stages I-IV correspond to early adenomas with sizes of $3 \mathrm{~mm}, 5 \mathrm{~mm}, 7 \mathrm{~mm}$, and $9 \mathrm{~mm}$, respectively. Stage V corresponds to an adenoma $\geq 1 \mathrm{~cm}$ or an adenoma with advanced villous histology, and stage VI corresponds to an adenoma of size $>2 \mathrm{~cm}$.

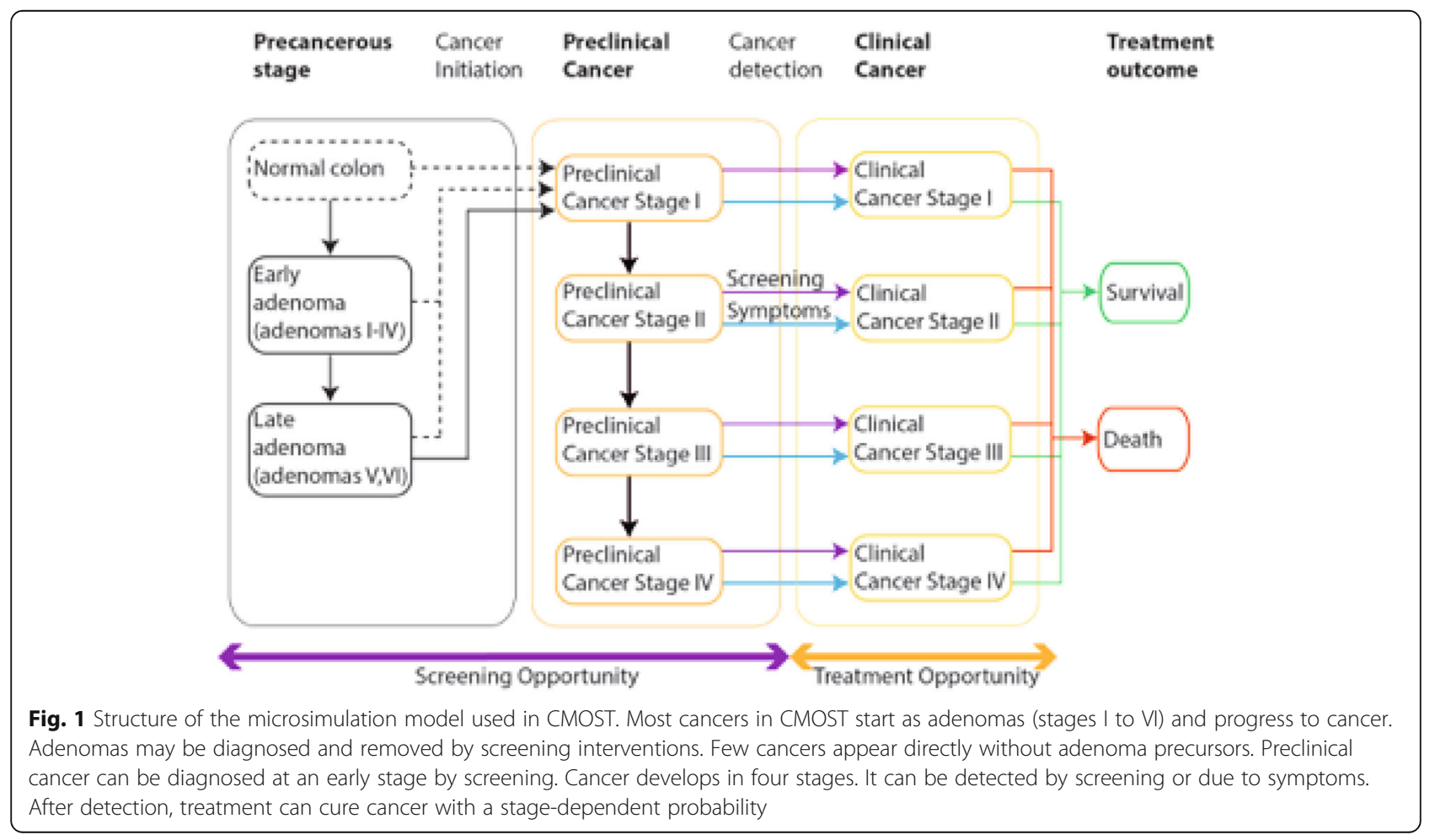


Table 1 Pseudocode for the CMOST program flow implemented in MATLAB

MAIN MODULE OF CMOST: individually tracks every individual in a population

Input:

- Model parameters determining natural history of CRC

- Settings for CRC screening and surveillance

Output:

- Death year, cause of death and years lost to CRC for each individual

- CRC incidence, prevalence, stages, mode of detection and outcome for each CRC and each individual

- Adenoma prevalence and adenoma stages for the whole population

- Usage of screening and surveillance interventions

- All CRC related costs

for Year $=1$ to 100 and if Alive $={ }^{\prime}$ YES'

for IndividualNumber $=1$ to NumberOflndividuals

call function AdenomaNaturalHistory(Record)

$\%$ - updates the natural history of adenomas and development to cancer

if (Screening.Mode $={ }^{\prime} \mathrm{ON}$ ' and Screening.Preference $={ }^{\prime} \mathrm{COLONOSCOPY}{ }^{\prime}$ and Screening.Year=Year) or

(Cancer.Symptomatic $=$ 'YES') or (FollowUp.Year $=$ Year)

$\%$ - if colonoscopy is a preferred screening option, or if cancer is symptomatic or for followup

call function Colonoscopy

if Treatment='ON' and (Year-Cancer.DetectionYear) $>5$ years

Treatment='OFF'

elseif Treatment='ON' and (Year-Cancer.DetectionYear) $<5$ years

call function AddCost(Treatment='ON')

if rand $>$ DeathFromCancer.Chance

Alive.Cancer=' $\mathrm{NO}^{\prime}$

DeathFromCancer.Year $=$ Year $\%$ - used for treatment costs in the last year before death from cancer

elseif rand > DeathFromNaturalCauses.Chance

Alive $={ }^{\prime} \mathrm{NO}^{\prime}$

end IndividualNumber

end Year

function AdenomaNaturalHistory(Record)

$\%$ - new adenoma(s) appear (influenced by age, gender, colon location, individual risk)

Record. $($ AdenomaStage $=I)=$ Record. $($ AdenomaStage $=I)+$ ChanceOfNewAdenoma

$\%$ - adenoma progression (influenced by age, gender, colon location, individual risk)

Record. (AdenomaStage $=\|$ to $\mathrm{Vl})=$ Record. $($ AdenomaStage $=\|$ to $\mathrm{VI})+$ AdenomaStageProgression

Record.CancerStage $(1)=$ Record.CancerStage $(I)+$ AdenomatousCancer(FromAdenomaStage $=\mathrm{VI}$ )

+ FastCancer(FromAdenomaStage $=$ I to $V)+$ DirectCancer(FromNoAdenoma)
Table 1 Pseudocode for the CMOST program flow implemented in MATLAB (Continued)

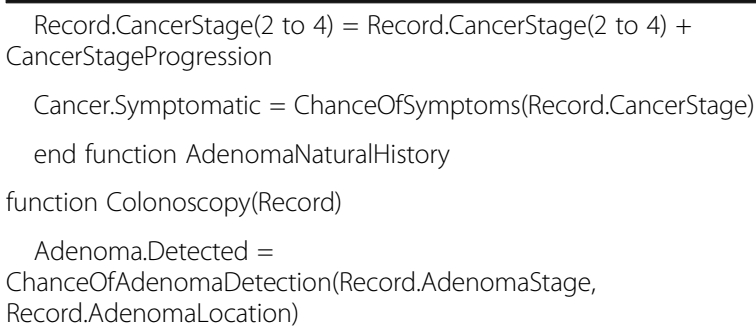

Cancer.Detected $=$ ChanceOfCancerDetection(Record.CancerStage, Record.CancerLocation)

if Cancer.Detected $={ }^{\prime}$ YES'

Cancer.DetectionYear $=$ Year

Record.Cancer $=0 \%$ - assume curative treatment has been performed

Treatment $=$ 'ON'

elseif Adenoma.Detected $=$ 'Yes'

Polypectomy $={ }^{\prime} Y E^{\prime}$

Record.Adenoma $=0 \%$ - remove the adenomas by polypectomy

Adenoma.Detected $=0$

FollowUp.Year $=$ RecommendedYear \% — recommend followup according to guidelines

if rand > ChanceOfComplication

Complications $=$ 'YES'

If rand $>$ ChanceOfDeathFromComplications

Alive $={ }^{\prime} \mathrm{NO}^{\prime}$

call function AddCosts(Colonoscopy, Polypectomy, Complications)

end function Colonoscopy

function AddCosts

Cost $=$ Cost + if(Colonoscopy $\left.={ }^{\prime} Y E^{\prime}\right) \times$ InputCost.Colonoscopy +

and in the last year of death from cancerif(Polypectomy $=$ 'YES') $x$ InputCost.Polypectomy +

and in the last year of death from cancerif(Complications $=$ 'YES') $\mathrm{X}$ InputCost.Complications +

and in the last year of death from cancerif(Treatment $=$ ' $O N$ ' $) \times$ InputCost.TreatmentCost(Year - Cancer.DetectionYear)

and in the last year of death from cancer\% - the treatment cost is divided into 3 stages: initial, continuing and last year costs for the first quarter, upto 5 years and in the last year of death from cancer

end function AddCosts

CMOST tracks the history of a general population from birth until death or a maximum age of 100 years. Adenoma initiation, progression to advanced adenoma and cancer, cancer progression, screening, and surveillance are all modeled in time increments of 3 months.

\section{Choice of parameters}

The relevant parameters used for the description of the natural history of CRC are summarized in Table 2, and their values are provided in the supplementary 


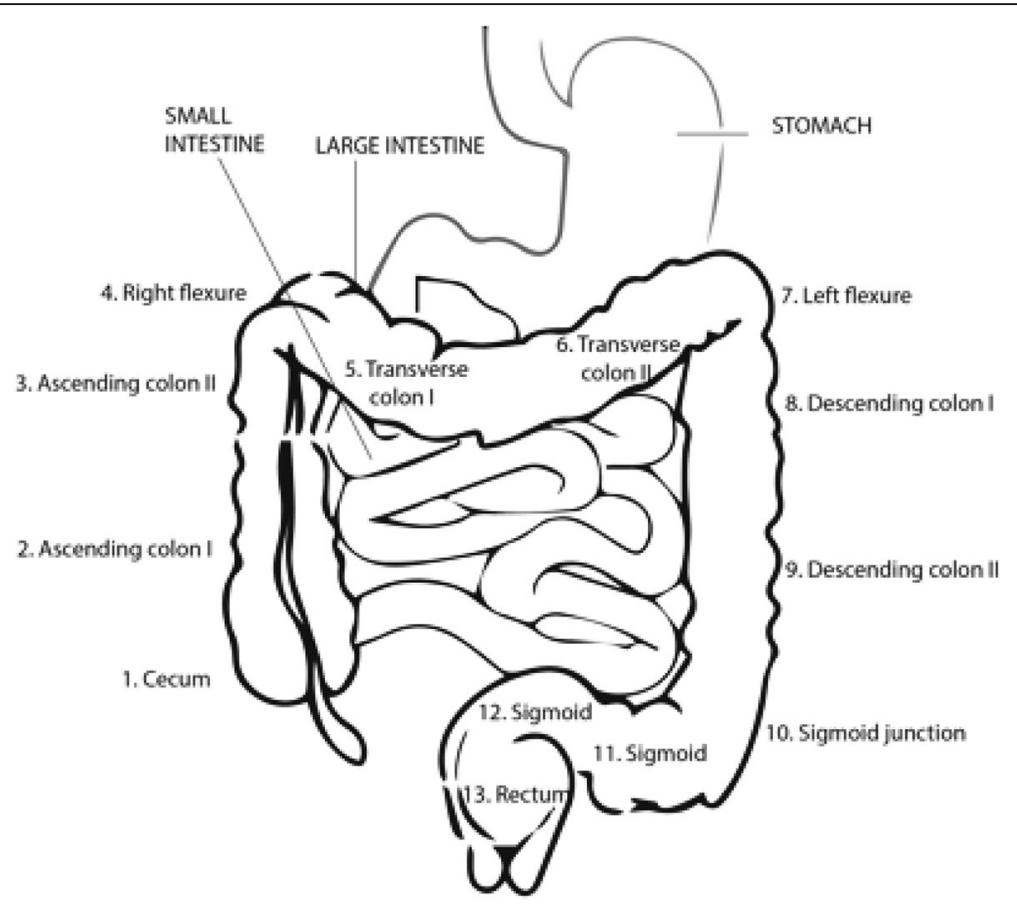

Fig. 2 Location of adenomas and cancer within the colon. The large intestine has been divided into 13 relevant segments. The CMOST model assumes location dependence for the progression and detectability of adenomas and cancers. Colonoscopy reaches the cecum in $95 \%$ of cases. Rectosigmoidoscopy is easier to perform but limited in scope and meant to reach the left flexure in the majority of cases

information. Some of these parameters depend on age and hence are defined by a function, for instance the age-dependent adenoma initiation risk of an individual is assumed to be a sigmoidal function. The sigmoid function was chosen since the benchmark parameter (i.e. age-dependent adenoma prevalence) also follows a sigmoidal function and can thus be conveniently reproduced. The model parameters age dependent early/ advanced adenoma progression are described by Gaussian functions, since these functions provide maximal flexibility allowing for a monotonously increasing or decreasing curve or a curve with a maximum.

Other model parameters are assumed to be stochastic and hence are defined by an assumed probability distribution. In this case the value for the individual or the adenoma is drawn randomly from the respective distribution in each simulation run. The parameter will then remain constant throughout the lifetime of the individual or adenoma. Examples include the risk for new adenomas specific for an individual or a progression risk specific for a given adenoma (see below). Other parameters, e.g., the relative risk of males versus females, are scaling factors for the respective condition.

Please note that our parameters cannot be directly compared to parameters from existing microsimulation models since different strategies for the description of the natural history of CRC were used [31]: For MISCAN, the duration of an adenoma in a given state (not adenoma progression rates) are used to describe the natural history of CRC and duration of adenomas are drawn from an exponential distribution. For CRC-Spin, adenoma growth follows a Janoschek growth model and CRC growth an exponential model. Finally, in SimCRC describing adenoma initiation and progression follow logistic regression curves, resembling our description of the natural history of CRC closest. It should be noted that due to the limited understanding of the natural history of CRC, the best mathematical descriptions of adenoma and CRC initiation and progression cannot be determined.

\section{Simulating the natural history of CRC}

At birth, an individual adenoma risk is assigned to each individual, which defines high or low CRC risk for this individual. At each time increment we decide about initiation of an adenoma, at random, considering both the individual adenoma risk and the age dependent adenoma risk. Upon adenoma initiation, an adenoma specific progression risk will be assigned to each adenoma. A high risk defines a quickly progressing aggressive adenoma, whereas a low risk defines a benign adenoma.

At each time increment, each adenoma might undergo one of the following changes: i) Adenoma progression: the risk for progression is determined by the product of an age-dependent adenoma progression risk, the 
Table 2 Description of the different parameters used in CMOST model. The procedure for calculating the parameters is described in the supplementary information

\begin{tabular}{|c|c|c|}
\hline Model parameter & Properties & $\begin{array}{l}\text { Functional form } \\
\text { (where } \\
\text { applicable) }\end{array}$ \\
\hline Age-dependent adenoma initiation risk & Defined by a sigmoidal function. & $\frac{a_{0}}{1+\exp \left(-\left(a_{1} y-a_{2}\right)\right)}$ \\
\hline Age-dependent early adenoma progression risk & Defined by a Gaussian function. & $\begin{array}{l}b_{0} \exp \left(-\left(b_{1} y-\right.\right. \\
\left.\left.b_{2}\right)^{2}\right)\end{array}$ \\
\hline Age-dependent advanced adenoma progression risk & Defined by a Gaussian function. & $\begin{array}{l}c_{0} \exp \left(-\left(c_{1} y-\right.\right. \\
\left.\left.c_{2}\right)^{2}\right)\end{array}$ \\
\hline Individual adenoma risk & $\begin{array}{l}\text { Cumulative density function describing the relationship between } \\
\text { population proportions and individual risk for appearance of new } \\
\text { adenomas. Defined by automatically calibrated anchor points. }\end{array}$ & \\
\hline Early and advanced adenoma specific progression risk & $\begin{array}{l}\text { Cumulative density function describing the relationship between } \\
\text { early or advanced adenoma proportions and adenoma specific } \\
\text { progression risk. Defined by manually calibrated anchor points. }\end{array}$ & \\
\hline $\begin{array}{l}\text { Correction factors male/female for i) adenoma initiation ii) } \\
\text { early adenoma progression iii) advanced adenoma } \\
\text { progression }\end{array}$ & Constant & \\
\hline $\begin{array}{l}\text { Correction factor rectum/colon for i) early adenoma } \\
\text { progression ii) advanced adenoma progression }\end{array}$ & Constant & \\
\hline Adenoma stage-specific progression risk & $\begin{array}{l}\text { Constant. One value for each adenoma stage } 1-6 \text {, enables fine } \\
\text { tuning of the distribution of adenoma stages } 1-6\end{array}$ & \\
\hline Adenoma stage-specific regression risk & $\begin{array}{l}\text { Constant. One value for each adenoma stage (1-6 highest for } 1 \text {, } \\
\text { lowest for } 6 \text { ); not calibrated }\end{array}$ & \\
\hline Adenoma stage-specific cancer risk & $\begin{array}{l}\text { Constant. One value for each adenoma stage ( } 1 \text { to } 5 \text { ), calibrated } \\
\text { to match prevalence of cancer in adenomas }\end{array}$ & \\
\hline Location specific direct cancer risk & $\begin{array}{l}\text { Constant. One value for each colon segment 1-13 (calibrated to } \\
\text { match CRC incidence reduction in rectosigmoidoscopy CRC } \\
\text { prevention study) }\end{array}$ & \\
\hline
\end{tabular}

progression risk of the given adenoma, the progression risk of the adenoma stage, and correction factors for female gender or rectal location if applicable. ii) Fast progression of adenoma to cancer: the risk of this event depends on the age-dependent advanced adenoma progression rate, the stage of the adenoma, gender, and location. iii) Adenoma regression: with very low probability, regression of an adenoma (or disappearance of a stage-I adenoma) is possible [32-36]; the risk of this event is defined by the adenoma stage.

Initiation of CRC can happen via three different pathways (Fig. 1): i) Progression of a stage 6 adenoma: this pathway accounts for approximately two thirds of all carcinoma in our microsimulation. ii) Fast progression of a smaller adenoma (stage 1-4) as described above. iii) Direct cancer without adenomatous precursors; such a pathway was introduced to match data from randomized endoscopic CRC prevention trials [12, 15, 37, 38] regarding prevention of right-sided adenomas after rectosigmoidoscopy screening. This direct cancer pathway represents cancers with precursors difficult or impossible to detect by colonoscopy. These cancers occur preferentially within the right colon and the direct cancer pathway reflects the reduced efficacy of CRC prevention by colonoscopy regarding right-sided CRC $[39,40]$.

At initiation of a cancer a sojourn time, i.e., the time from the initiation of the cancer to it becoming symptomatic, is assigned to the cancer. The sojourn time is drawn from a normal distribution of values with a mean of 3 years $[8,41]$ and a standard deviation of 0.5 years. The stage at which symptoms will appear is chosen to reflect the stage distribution of symptomatic cancers. The time spent in each stage is also predefined for each cancer to reflect the stage distribution of cancers detected during colonoscopy screening [42].

After sojourn time a carcinoma will become symptomatic, triggering a diagnostic colonoscopy. Upon detection of a cancer, survival time will be defined, following published survival rates for the respective CRC stage [43]. Death from CRC is restricted to 5 years after CRC diagnosis.

In our microsimulation death results from colon cancer, medical interventions, or from other age-dependent causes of mortality according to the 2008 US Life Table Data [44]. An individual patient who dies of CRC is 
followed in the simulation until he would have died from other causes.

\section{Simulation of CRC screening}

CMOST supports simulation of CRC screening and detection by colonoscopy. For each colonoscopy, the amount of colon visualized is drawn from a specified distribution, with $95 \%$ of all colonoscopies reaching the cecum on average. For each adenoma, our microsimulation will determine whether the respective lesion will be detected during a given intervention [45]. The probability of detection depends on adenoma stage and whether the lesion resided within the visualized colon. CMOST also accounts for slightly reduced adenoma detection at the hepatic and splenic flexure. CMOST considers the following complications of colonoscopy: i) major bleeding (4 per 10,000), ii) minor bleeding (11 per 10,000), iii) mucosal burn (3 per 10,000) and iv) perforation (7 per 10,000) [46]. The probabilities of these complications are derived from published data and increase 2-fold after polypectomy [47].

Rectosigmoidoscopy is implemented similar to colonoscopy but with the limitation that the colon is visualized only from rectum to the left-flexure (on average 6 of the 13 segments of the colon). Due to poor bowel preparation and additional technical limitations, the probability to detect an adenoma is assumed to be lower than for colonoscopy (12.5\% lower probability of detection for advanced adenomas and $25 \%$ for early adenomas compared to colonoscopy). The risk of complications during rectosigmoidoscopy is much lower than for colonoscopy [48] and no lesions will be removed. Any lesion detected at this intervention will trigger a colonoscopy.

CMOST also supports additional screening interventions including fecal occult blood test (FOBT) or immune -FOBT. The parameters of all screening intervention as well as adherence to screening and follow-up colonoscopy can be freely adjusted. CMOST allows for the definition of a screening plan with a starting date, a finishing date, an adherence rate and a screening interval.

\section{Cost calculations}

Our model includes costs for screening colonoscopy, potential polypectomy, and procedural complications. In our cost calculations, treatment costs during 5 years after cancer diagnosis are considered. Treatment is divided into three phases: The initial care phase lasting 3 months includes cancer detection, surgery and radiation as well as chemotherapy in selected situations depending on the stage and location of the carcinoma. In case of death from CRC, the phase of terminal care lasts up to 12 months and includes additional palliative surgery in 50\% and palliative chemotherapy in all patients. The period of continued treatment with monitoring and consultation visits spans from the end of the initial phase to the beginning of the last year or the end of treatment. Screening and treatment costs (Additional file 1: Table S1) are based on the Diagnosis Related Group (DRG) codes for the outpatient setting and Current Procedural Terminology (CPT) codes for the inpatient setting, using the corresponding Medicare reimbursement schedule (http:// www.cms.gov). Average national US payments for the year 2012 are used in the present calculations. Costs and life years gained are computed via comparison to those that accrue in the non-screening scenario. Both, costs and LYG are discounted by a $3 \%$ annual discount rate. A list of costs used for our study is provided in Additional file 2: Tables S7 and Additional file 3: Table S8.

\section{Automated parameter calibration}

CMOST supports automated calibration of model parameters to meet epidemiological benchmarks regarding the natural history of colorectal cancer. To calibrate our model to the US American population, we used published age- and sex-specific adenoma prevalence rates as benchmarks [18, 49-51]. Carcinoma incidence and mortality, as well as separate colon and rectum cancer incidence rates, are modeled according to data from the Surveillance Epidemiology and End Results (SEER) database for 2005-2009 [52]. The stage distributions of symptomatic and asymptomatic cancers also follow published analyses [11, 53]. Altogether we used 105 benchmarking data points covering various quantities predicted by the model for parameter estimation. A full list of all benchmarks is provided in Additional file 1: Table S1. Our strategy for automated parameter calibration is explained in detail in Section V of the Supplement. Briefly: we divide the natural history of CRC into four steps (I: early adenoma, II: advanced adenoma, III: cancer, IV: direct cancer) and perform calibration of the group of parameters relevant for each of these steps sequentially. Our implementation of the natural history of CRC has been designed in a way that for each calibration step a lower step will influence the readouts of all higher steps but not vice versa. For instance, adjusting parameters in step 1 to increase the incidence of early adenomas by $10 \%$ will also change the incidence of advanced adenomas by $10 \%$. Vice versa, modifying parameters of step 2 to increase adenoma progression to increase advanced adenoma incidence by $10 \%$ will affect early adenoma prevalence to a lesser degree: Faster early adenoma progression will decrease early adenoma prevalence since some 
early adenomas will be advanced adenomas now. However, the decrease will only be approximately $1 \%$ since prevalence of early adenomas is 10 times higher than for advanced adenomas. For these reasons, all four steps can be considered independent calibration steps and are performed sequentially and sequential calibrations yields in satisfactory results.

However, practical tests showed that even better fitting could be achieved by a final calibration step which simultaneously readjust parameters for step 2 and 3 simultaneously. Furthermore, our program provides the option to automatically perform parameter calibration of steps 1-3 sequentially, followed by a fine-tuning of parameters in steps 2 and 3 as a single procedure (for instance on a cluster computer). Details of these procedures are explained in the Manual.

For each calibration, we used a heuristic greedy algorithm followed by Nelder-Mead optimization [54] to minimize the squared error between the benchmark values and the corresponding model predictions for parameter estimation. Automated parameter calibration is required only when the benchmarks of the natural history are changed or when different possibilities for the adenoma dwell time within the same natural history are to be explored.

\section{Implementation of CMOST}

Details of all features of CMOST can be found in the accompanying manual. The software is available under a GNU general public license and can be downloaded at https://gitlab.com/cmostmodel/CMOST. CMOST has been implemented in Matlab ${ }^{\circ}$ Time critical calculations were implemented using the Coder module of Matlab, resulting in a 20x improvement in performance.

All functionality of CMOST is available via several intuitive graphical user interfaces. All relevant parameters, including values for all variables describing the natural history, specifications for CRC screening, and a screening plan, can be saved and loaded as a settings file. CMOST can be run on a desktop computer and we provide basic support in the form of scripts for running it on LINUX compute clusters.

CMOST produces the following output files after each calculation: i) a Matlab data file with the raw computation results, which can be used for further calculations in Matlab; ii) several PDF files containing plots of all relevant variables that describe the prevalence and distribution of adenomas, advanced adenomas, and CRC, relative to the benchmarks used; iii) an Excel file with a summary of the CMOST simulation run.
The accurate determination of the optimal age for reduction in incidence, mortality, or cost per life year gained required calculations on population sizes of up to 10 million. The calculations are repeated for each condition, e.g., the year of recommended screening colonoscopy. Each of these cases is, in principle, a single processor job. However, in order to effectively handle the memory available on each processor, the calculation on the population of 10 million was subdivided into calculations with 100,000 individuals on a high-performance LINUX computer cluster.

\section{Results}

\section{A new microsimulation model for the natural history of} CRC

We developed CMOST, a microsimulation model to simulate CRC progression and the effect of CRC screening (Fig. 1). In our model carcinoma develops via early and advanced adenoma precursors. Altogether, 6 adenoma stages and 4 carcinoma stages are considered in the model. Most of the transition towards pre-clinical cancer occurs via the adenomatous pathway, advancing through the 6 successive stages of adenoma progression. With lower probability, pre-clinical cancer can also start from any of the adenoma stages or even from seemingly normal colon mucosa.

Our model accounts for the gender- and agedependent risks of adenoma development $[5,18,50$, $55]$ as well as the presence of multiple adenomas. Each adenoma will be assigned one of 13 locations within the colon, reflecting the distribution of adenoma lesions within the colon [24]. In our model, rectum adenomas progress faster compared to colon adenomas to achieve the expected proportion of rectum cancers of all CRC [56]. Each individual within the simulated patient population is assigned an individual adenoma risk. The distribution of these risks within the whole population is calibrated to match the frequency of multiple adenomas [49] and also matches the frequency of synchronous colorectal cancers of $3.5 \%$ of all CRC [57].

Adenoma dwell time indicates the time between appearance of an adenoma and transition to colorectal cancer. Since adenoma dwell time can only be estimated empirically with a broad margin of error, we considered three parameterizations of CMOST with dwell times of 8,13 , and 19 years, referred to as CMOST8, CMOST13, and CMOST19, respectively.

\section{Automated calibration of our model}

To calibrate our model for the North American or a similar Western population, published age- and sexspecific adenoma prevalence rates were used $[18,50$, 
55]. Carcinoma incidence and mortality, as well as separate colon and rectum cancer incidence rates, are modeled according to the data from the Surveillance Epidemiology and End Results (SEER) data base for 20052009 [52]. Altogether, 105 benchmarks were selected (Additional file 1: Table S1) and an automated calibration strategy was employed (see Additional file 4). A comparison with the benchmarks is performed at every iteration of the automated parameter calibration. The calibration is terminated when the comparisons are satisfactory, such as shown in Fig. 3. CMOST allows the flexibility of including a new set of CRC natural history benchmarks from a new population, and the four-steps of the automated parameter calibration can be repeated.

CMOST assumes a fraction of all cancers to appear without adenomatous precursors (referred to as direct cancer henceforward). Benchmarking for direct cancer was performed in an indirect way using data from a randomized rectosigmoidoscopy study with 170,432 individuals between 55 and 64 years of age [58]. After a onetime only rectosigmoidoscopy (visualizing the left-sided colon), individuals with positive findings are followed by a full colonoscopy, thus enabling CRC prevention on the left and the right side of the colon. Overall CRC incidence reduction was used as a benchmark to parameterize the rate of direct cancers (further details in Additional file 4).

\section{Simulation of CRC screening}

In our microsimulation, colonoscopy will either be performed according to a pre-defined screening schedule, for adenoma or cancer surveillance, or for the diagnosis of symptomatic cancer. Our implementation of colonoscopy accounts for varying probabilities of successful visualization of the hepatic and splenic flexures and imperfect detection of early and advanced adenomas and colorectal cancer [45, 59]. Complications of colonoscopy including major bleeding, which requires hospitalization; minor bleeding, which does not require hospitalization; perforation, which requires surgical treatment; and mucosal burn, are all accounted for [60]. We also simulated surveillance colonoscopies after the detection of a lesionaccording to current guidelines [61]. Other screening interventions, including rectosigmoidoscopy and fecal occult blood tests with various test parameters, have also been implemented. Our program can be used to assess effects of every CRC screening method including DNA stool tests, DNA blood tests or imaging based tests provided that information about test sensitivity, specificity, risks and the costs are available.

\section{Comparison of CMOST to randomized CRC screening trials}

To validate CMOST, we compared the predictions of our model to the independent results of a large randomized controlled trial of endoscopy for CRC prevention [37] (Table 3) that were not used for model calibration. In this study, the effect of one or two rectosigmoidoscopies in a combined study group of 77,445 individuals aged 55-74 years was tested over an 11.9-year follow-up period. While the study is similar to the one used for benchmarking the direct cancers [12], the study group is slightly larger, start and end times differ, and two endoscopies are used in a significant fraction of patients. Results of CMOST13 simulations compare well with the intention-to-treat analysis of the randomized study and its confidence intervals: CMOST13 predicts a similar overall incidence reduction (19.9\% predicted vs. $21 \%$ observed), left-sided CRC incidence reduction (24\% predicted vs. $29 \%$ observed) and overall mortality reduction (26\% predicted vs. $26 \%$ observed) [37]. Similar results were obtained using CMOST8 and CMOST19 (Table 3).


Fig. 3 Results of CMOST regarding adenoma and cancer epidemiology for the whole population (males and females). a: Early adenoma prevalence $\mathbf{b}$ : Advanced adenoma prevalence $\mathbf{c}$ : Cancer incidence. Literature-derived benchmarks (see Additional file 4) used for our microsimulation are indicated by black squares and a blue dashed line; results of CMOST13 calculations are shown as a black line and green-squares when within 20\% of benchmarks, and as red-squares otherwise 
Table 3 Effects of rectosigmoidoscopy screening for CRC prevention combined with 11.9-year follow-up (intention to treat analysis according to the randomized controlled study by Schoen et al. [37])

\begin{tabular}{llllll}
\hline & & Schoen et al. [37] with 95\% Cl & CMOST8 & CMOST13 & CMOST19 \\
\hline Incidence reduction & All CRC & $21 \%(28-15)$ & $20 \%$ & $20 \%$ & $20 \%$ \\
& Right-sided CRC & $14 \%(3-24 \%)$. & $15 \%$ & $7 \%$ & $24 \%$ \\
& Left-sided CRC & $29 \%(20-36)$. & $21 \%$ & $26 \%$ & $26 \%$ \\
Mortality reduction & & $26 \%(13-37)$ & $23 \%$ & $23 \%$ \\
\hline
\end{tabular}

We also simulated other smaller CRC prevention studies (Additional file 5: Table S9), and the simulation results were also in agreement with the observed data $[15,38]$. We conclude that CMOST reflects the natural history of CRC well and reliably predicts the outcome of randomized rectosigmoidoscopy screening trials.

\section{Comparison of CMOST with existing CRC simulation models}

Predictions of CMOST were compared to those of other microsimulation models describing the natural history of CRC, namely MISCAN, CRC-SPIN, and SimCRC, following two previously published comparative studies [7, 62]. In a first comparison, a hypothetical perfect screening intervention, removing all lesions within the colon of each individual (early adenomas, advanced adenomas, and cancers) at the age of 65 was modeled (referred to as maximum clinical incidence reduction in the original publication [7]). All CRC detected after the age of 65 would thus be newly developed lesions. CMOST8, CMOST13, and CMOST19 differ slightly regarding future CRC incidence (Fig. 4). Predictions of CMOST8 strongly resemble predictions of MISCAN while CMOST13 and CMOST19 predict a lower CRC incidence 20 years after the hypothetical intervention (Fig. 4). Additional detailed comparisons between all microsimulation

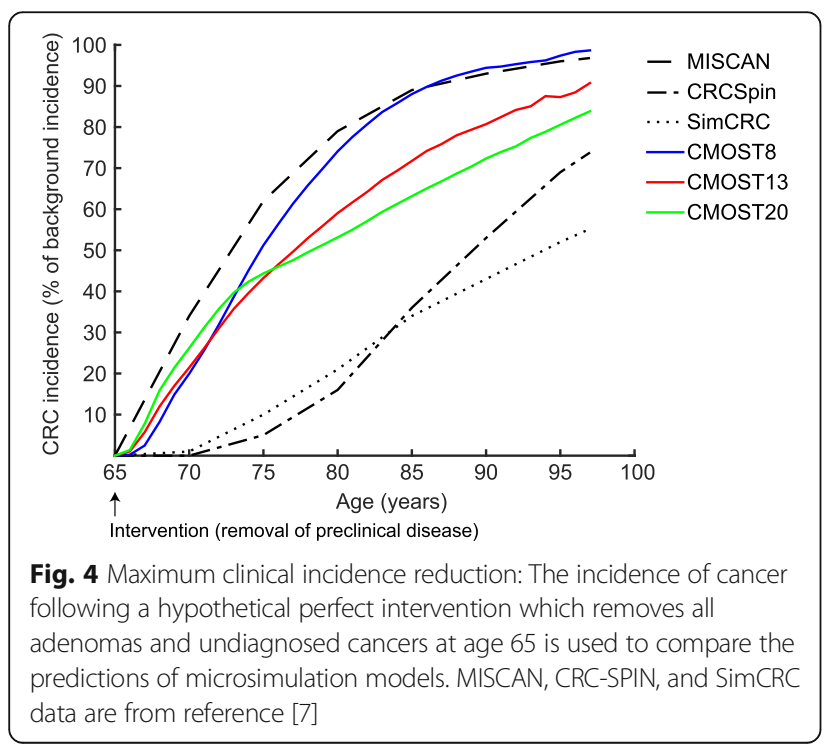

models are provided in Additional file 6 Tables S2, Additional file 7: Table S3, Additional file 8: Table S4, Additional file 9: Table S5 and Additional file 10: Table S6.

We also tested predictions of CMOST with regard to the effects of colonoscopy on colon cancer incidence and mortality (Table 4) and found that CMOST was closest to the MISCAN model. Since for all versions of CMOST, i.e., CMOST8, CMOST13, and CMOST19 the direct cancer rate was calibrated to achieve an identical incidence reduction as a randomized rectosigmoidoscopy study, predictions of all CMOST versions were very similar. Thereby, the fraction of direct cancer is lowest for CMOST8, intermediate for CMOST13, and highest for CMOST19. Effects of a longer dwell time (19 years for CMOST19, 13 years for CMOST13, leading to longer lasting effects of adenoma removal than for CMOST8) will be offset by a higher fraction of direct cancers which cannot be prevented by CRC screening. Without this calibration step (with identical direct cancer rates) incidence reduction for CMOST19 would be highest, intermediate for CMOST13, and lowest for CMOST8 (data not shown).

On a different note, CMOST was calibrated to reflect contemporary CRC incidence, whereas MISCAN, CRCSPIN, and SimCRC reflect the higher CRC incidence of 1977 (before the onset of CRC screening). This difference explains some of the discrepancies within Table 4 (compare "CRC cases prevented" or "colonoscopies per life year gained" for CMOST and the established models). We also simulated the effect of FOBT screening regarding CRC incidence and mortality reduction. The results obtained by CMOST are similar to those from SimCRC and MISCAN according to published data [63] (Additional file 9: Table S5, Additional file 10 Table S6). Taken together, predictions by CMOST regarding the natural history of CRC development and CRC screening are comparable to published microsimulation tools. All versions of CMOST resulted in similar predictions for various read outs, and predictions of CMOST strongly resemble those of MISCAN.

\section{Defining the optimal time point for colonoscopy screening}

We tested the optimal application of colonoscopy in a scenario allowing for a single screening colonoscopy 
Table 4 Comparison of CMOST with other microsimulation tools. Screening colonoscopies were performed at 10-year intervals between ages 50 and 75 years. Results of the microsimulation tools MISCAN, CRC-SPIN, and SimCRC were taken from reference [7]. Numbers indicate percentage reduction or the number of additional colonoscopies or CRC cases as indicated per 1000 individuals. For CMOST, the numbers of the baseline scenario assuming a dwell time of 13 years (CMOST13) are given; numbers in parenthesis indicate results obtained by using CMOST8 and CMOST19

\begin{tabular}{|c|c|c|c|c|}
\hline & MISCAN & CRC-SPIN & SimCRC & CMOST13 (CMOST8, CMOST19) \\
\hline Incidence reduction & $52 \%$ & $91 \%$ & $82 \%$ & $53 \%(55,48)$ \\
\hline Mortality reduction & $65 \%$ & $92 \%$ & $84 \%$ & $61 \%(65,55)$ \\
\hline Life years gained & 207 & 260 & 327 & $142(155,121)$ \\
\hline Screening colonoscopies & 2288 & 2580 & 2574 & $2373(2304,2289)$ \\
\hline Surveillance colonoscopies & 1715 & 1341 & 1609 & $1311(1222,1251)$ \\
\hline Total colonoscopies & 4002 & 3921 & 4184 & $3600(3538,3558)$ \\
\hline CRC cases prevented & 30 & 56 & 54 & $27(28,23)$ \\
\hline CRC mortality cases prevented & 19 & 25 & 30 & $12(13,10)$ \\
\hline Colonoscopies per case prevented & 135 & 70 & 77 & $132(124,155)$ \\
\hline Colonoscopies per LYG & 19 & 15 & 13 & $25(22,29)$ \\
\hline
\end{tabular}

during the lifetime of a patient. We compared incidence and mortality of CRC to a scenario without screening (Fig. 5a, b). For all versions of CMOST, incidence reduction from colonoscopy increases with age at first colonoscopy up to an optimum at approximately 58 years. Both the extent of the benefit conferred by colonoscopy (incidence reductions in CMOST8, CMOST13, CMOST 19 are 36\%, 36\%, and 35\%, respectively) and the optimal time point for colonoscopy were comparable for all simulation models tested $(59,58$, and 56 years; Fig. 5a). Similarly, the mortality reductions predicted by CMOST8, CMOST13, and CMOST19 are 43\%, 40\%, and $39 \%$, respectively, all at the optimal ages of 61,60 , and 50 years (Fig. $5 b$ ).

We also calculated life years gained (LYG) and costefficiency of colonoscopy. Following current practice in health economics, LYG and costs were compared to the standard scenario without screening. Differences were

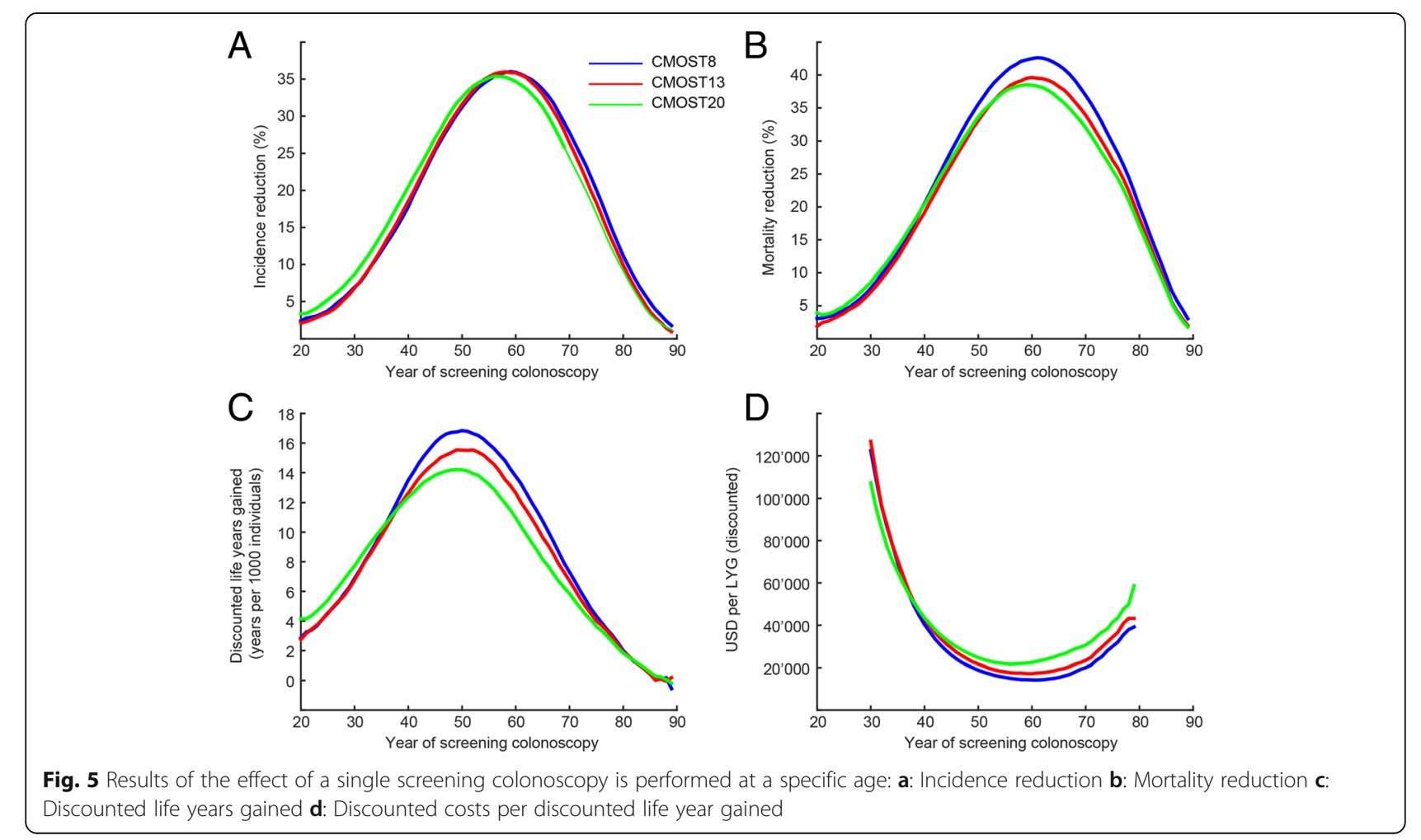


discounted by 3\% per year (Fig. 5c). Since prevention of a carcinoma in a young patient will save more life years compared to an older individual, the optimum for LYG is at a lower age than for incidence and mortality reduction. For CMOST8, CMOST13, and CMOST19, 17, 16 and 14 discounted years per 1000 persons, respectively, were obtained. An optimum was reached at similar time points, (age 50, 49, and 49 years, respectively). Without discounting, the number of LYG would be considerably higher (102, 94, and 85 years per 1000 individuals) at somewhat later time points $(53,54$ and 53 years, respectively).

Cost efficiency was estimated by dividing discounted costs in USD by discounted LYG. By common conventions in health economics, any intervention with costs less than 100,000 USD per LYG is considered costeffective. In our calculations, the optimal values of costefficiency for a single screening colonoscopy were $\$ 14,209, \$ 17,211$, and $\$ 21,698$ USD/LYG, and thus costeffective for all three versions of CMOST. As for incidence and mortality reduction, the optimum costeffectiveness is attained at a similar age in all versions of CMOST tested, i.e., for CMOST8, CMOST13, and CMOST19, at 61, 59, and 56 years, respectively (Fig. 5d).

\section{Discussion}

We have introduced CMOST, a new microsimulation model for the natural history of CRC. CMOST reflects all basic characteristics of the natural history of CRC, shows comparable characteristics to published models of CRC microsimulation, and can reproduce the results of a large randomized endoscopic screening intervention.

Our tool offers several benefits to the user. First, our model is publicly available under a GNU General Public License. This will enable independent reproduction of predictions and advancement of the model and its implementation. Furthermore, publication of all details of our microsimulation will enable scrutiny and a detailed discussion regarding all relevant aspects of CRC simulations. We hope that future extensions of CMOST will help increasing the validity of simulation results and further improve the in silico design of CRC screening strategies. Additional advancements of our model and its implementation include calibration against results of a recently published endoscopic screening trial, which had not been possible at the time of the development of previous models. The transparency of benchmarking and automated calibration of CMOST parameters will facilitate further improvements to the model. Repeated convergence to the same set of parameters for different initial parameter guesses suggests a well-defined optimum in parameter space of CMOST.

We believe that many aspects of the CMOST implementation will benefit future users of the tool. CMOST can be run using a graphical user interface, which allows adjustment of all relevant parameters. Time critical routines are accelerated using the coder option of Matlab, with a $20 \mathrm{x}$ improvement in performance. A simulation of 100,000 individuals implemented in Matlab R2015 and executed on a $2.5 \mathrm{GHz}$ processor, uses $1 \mathrm{~GB}$ RAM and typically finishes in less than $1 \mathrm{~min}$. In addition, CMOST supports usage of a high-performance compute cluster for either the simulation of large populations or detailed explorations. There is no agreement as to what level of detail is necessary for a CRC microsimulation to be both accurate and practical. We attempted to implement CMOST as a detailed microsimulation. For instance, 13 different locations of a lesion within the colon are supported and many details of CRC pathogenesis and screening can be adjusted according to these locations.

Since the true value (or probability distribution) of adenoma dwell times is unknown, we calibrated versions of CMOST with median dwell times of 8, 13, and 19 years (CMOST8, CMOST13, CMOST19). Predictions of CMOST8, CMOST13, and CMOST19 were similar and showed resemblance to MISCAN. Due to our calibration process, the longer dwell time of CMOST19 (leading to longer lasting effects of adenoma removal) would be offset by a higher rate of direct cancer which cannot be prevented by endoscopic screening. As in previous studies, when MISCAN, CRC-SPIN, and SimCRC were frequently tested in parallel to increase robustness of predictions, different versions of CMOST can be run for increased robustness of predictions. Our data confirm adenoma dwell time as a critical parameter for the natural history of CRC; however, we demonstrate that after calibration for relevant clinical endpoints, similar results can be achieved with models using considerably different dwell times.

Currently available epidemiological data do not allow for distinguishing whether CMOST8, CMOST13, or CMOST19 reflects the natural history of CRC best. We were unable to calibrate models with less than 8 years of adenoma dwell time suggest that this parameter is bounded, at least for a given set of benchmarks. Predictions of all CMOST versions regarding overall CRC incidence and mortality reduction and protection from leftsided CRC were well within the confidence interval of a large endoscopic CRC prevention trial. In the future, results of colonoscopy CRC prevention trials will further inform microsimulation models regarding the prevention of right-sided CRC.

According to the standard health economic definitions, an intervention is considered cost-effective if the costs remain below 100,000 USD per life year gained $[64,65]$. Colonoscopy screening in that sense is a costeffective intervention. In agreement with the results of 
numerous previous microsimulations [66, 67], screening costs of a single screening colonoscopy remains below the accepted limit of 100,000 USD per life years gained. Our results also largely agree with an earlier study based on a Markov model, recommending a single colonoscopy at age 60 years as a highly cost-effective screening strategy [68]. Several previous computational studies reported an even better cost-effectiveness than our study, and some studies even predicted CRC screening to be cost saving [66]. These discrepancies are largely due to different cost assumptions. Past studies used Medicare data from the years 1999-2003 [46, 69] with some studies even considering the cost of death due to reasons other than cancer. In contrast, the cost assumptions in the present study were based on the Medicare reimbursement schedule from 2012. Therefore, in previous microsimulations screening tended to be cheaper or treatment costs were higher compared to ours, making our cost calculations conservative.

Our tool provides flexibility to easily implement and evaluate basically all CRC screening options provided information regarding test sensitivity, specificity, risks and costs are provided. Examples include DNA based serum and stool tests or imaging based screening methods. Furthermore, big efforts are currently underway to ensure the quality of colonoscopy as the most frequently used CRC screening tools: Modifications in the procedure such as endoscope inversion in the cecum to improve adenoma detection there, adjustments in bowel preparation to improve "cleanliness" of the colon, enforcing a retraction time of colonoscopy of at least $6 \mathrm{~min}$ have been shown to increase adenoma detection rates. However, these procedures have direct or indirect costs linked to a longer time of the investigation, higher demands for documentation or patient and physician education. Our tool will enable to assess the benefits of each modification and put it into perspective by comparison with increased costs. An incremental cost-effectiveness ratio of 100 '000 USD per LYG will determine, whether any modification should be recommended or advised against.

Our microsimulation has also several inherent limitations: i) Key parameters of the natural history of CRC are unknown. This is especially relevant for the adenoma dwell time, the time from the appearance of an adenoma until its transition to preclinical CRC. However, we provide three versions of CMOST spanning a wide range of reasonable assumptions for this parameter, enabling sensitivity analyses. ii) The serrated adenoma pathway is not explicitly considered by our model. While implementation of an additional adenoma path with preferential distribution of serrated adenoma lesions in the right colon and lower detectability by colonoscopy is technically feasible, most characteristics of serrated adenoma epidemiology remain unknown, reducing model calibration to guess work. However, our model allows for carcinoma directly developing from normal mucosa with a right-colonic preference implemented, at least partially accounting for the serrated adenoma path. iii) Our model does not account for benign non-adenomatous polyps. iv) CMOST has been calibrated to contemporary CRC incidence data. This contrasts previous models, which were calibrated using data of the year 1977, before the introduction of CRC screening. For these reasons, CRC incidence for MISCAN, SimCRC, and CRC-SPIN remains higher than for CMOST. Several studies indicate that the decrease in CRC incidence observed within the last decades is not only due to CRC screening but might also be due to usage of non-steroidal antirheumatic drugs [70] or changes in smoking [71] and nutrition [72]. These different effects cannot be disentangled.

\section{Conclusions}

In summary, we have introduced CMOST, a new CRC microsimulation tool, freely available under a general public license. Predictions of our model regarding the natural history of CRC and CRC screening were similar to predictions of published CRC microsimulation tools and an endoscopic CRC screening study, confirming the validity of our model. CMOST predicts CRC screening by colonoscopy to be highly cost-efficient with an optimal time point of a single colonoscopy for maximum costefficiency of approximately 61 years. Our computations confirm adenoma dwell time as a critical parameter for CRC microsimulation models; however, similar results for various effects of colonoscopy screening including incidence reduction, cost-efficiency, and optimal time points were obtained with a dwell time of 8,13 , and 19 years.

Our tool enables assessment of many practical questions in current gastroenterology. For instance, cost-effectiveness of new CRC screening approaches, or of incremental changes of existing screening approaches such as improvements in colonoscopy screening by improved bowel preparation or physician training can be assessed. Additionally, cost-effectiveness of CRC screening at extremes of age, comorbidities or risks could be addressed. Basically, most practical question regarding screening or treatment of CRC which can be expressed in terms of risks and costs, our tool should be able to answer, perhaps after modification of the code. Thereby, the flexibility of our model enables adaptation also to countries with different CRC epidemiology such as in developing countries. Additional case studies will improve the practical value of our program in the future.

The main limitations of our tool is the limitation in our knowledge of CRC, making assumptions for adenoma dwell time and the distribution of risks for adenoma progression and initiation necessary. Furthermore, several aspects of the natural history of CRC such as the serrated 
adenoma pathway are not reflected by our program to limit complexity. Results of ongoing randomized CRC screening trials using colonoscopy (expected within 5-10 years) will improve the validity of predictions of our tool.

\section{Additional files}

Additional file 1: Table S1. All versions of CMOST were calibrated relative to the 9 categories indicated below (105 data points). For parameters with gender differences (early and advanced adenoma prevalence, cancer incidence) the parameter for the whole population was adjusted with a correction factor for males and females. For stage distribution of symptomatic and asymptomatic cancer the distribution of time spent in a given stage as well as the stage at which the cancer would be symptomatic was adjusted to yield in a sojourn time of 3 years and the indicated stage distributions (benchmarks 8 and 9). (DOCX 32 kb)

Additional file 2: Table S7. Costs of colonoscopy and its complications. (DOCX $13 \mathrm{~kb}$ )

Additional file 3: Table S8. Stage dependent costs of cancer treatment. (DOCX $13 \mathrm{~kb}$ )

Additional file 4: Manual and Literature. (DOCX $40 \mathrm{~kb}$ )

Additional file 5: Table S9. Simulation of rectosigmoidoscopy screening for prevention of CRC according to randomized controlled trials (intention to treat analysis). Modeling of these studies provides additional validation of our model. (DOCX $19 \mathrm{~kb}$ )

Additional file 6: Table S2. Comparison of CMOST with other microsimulation models [64]: Individuals with and without preclinical disease at age 55 (adenomas, undiagnosed cancer) were identified and the cancer rates over the next 20 years were compared. (DOCX 13 kb)

Additional file 7: Table S3. Comparison of CMOST models with other microsimulation models [64]: For cancer diagnosed at the indicated ages $(55,65$ or 75 years) the percentage of cancer developing over the last $\leq 10$ years or $\leq 20$ years before cancer diagnosis (i.e. adenomatous precursor present) is indicated. (DOCX $14 \mathrm{~kb}$ )

Additional file 8: Table S4. Comparison of CMOST models with other microsimulation models [64]: Adenoma dwell time cancer sojourn time and overall dwell time are indicated. Direct cancer was ignored for calculations of dwell time. (DOCX $14 \mathrm{~kb}$ )

Additional file 9: Table S5. Comparison of CMOST models with other microsimulation models [65]: Predicted incidence reduction of various screening interventions. (DOCX $13 \mathrm{~kb}$ )

Additional file 10: Table S6. Comparison of CMOST models with other microsimulation models [65]: Predicted mortality reduction of various screening interventions. (DOCX $13 \mathrm{~kb}$ )

\section{Abbreviations}

CMOST: Colon modeling with open source tool; CRC: Colorectal cancer; CRCSPIN: Colorectal cancer simulated population model for incidence and natural history; ICER: Incremental cost effectiveness ratio; LYG: Life years gained; MISCAN: Microsimulation screening analysis; SimCRC: Simulation model of colorectal cancer (SimCRC)

\section{Acknowledgements}

The authors would like to thank Stephan R. Vavricka, Christine Manser, Luc Biedermann, Patric Urfer, Roger Wanner, and Urs Marbet for stimulating discussions, and help with thorough literature research. We would like to thank Martin Fox, BSSE Basel and the management of the Brutus and Euler computer clusters at ETH Zurich for technical support.

\section{Funding}

This work was supported by Swiss Cancer League grant No. KFS-2977-082012 and the Helmut Horten Foundation.

Availability of data and materials

The proposed tool is available under https://gitlab.com/misselwb/CMOST

\section{Authors' contributions}

MP: Study design, data analysis and manuscript preparation; BL: Scientific concept, critical review of the manuscript; NB: Scientific concept, critical review of the manuscript; $\mathrm{HH}$ : data analysis and manuscript preparation, $\mathrm{PV}$ : data analysis and manuscript preparation, PB: Scientific concept, study design; AS: Scientific concept, study design, manuscript preparation; BM: Scientific concept, study design, data analysis and manuscript preparation. All listed authors have seen and have approved the final draft submitted.

\section{Competing interests}

The authors declare that they have no competing interests.

\section{Consent for publication}

Not applicable

Ethics approval and consent to participate

No human or animal data was used for this study, therefore ethics approval was not necessary.

\section{Publisher's Note}

Springer Nature remains neutral with regard to jurisdictional claims in published maps and institutional affiliations.

\section{Author details}

${ }^{1}$ Division of Gastroenterology, University Hospital Zurich (USZ), Rämistrasse 100, 8091 Zurich, Switzerland. ${ }^{2}$ Department of Biosystems Science and Engineering, ETH Zurich, 4058 Basel, Switzerland. ${ }^{3}$ SIB Swiss Institute of Bioinformatics, 4058 Basel, Switzerland. ${ }^{4}$ The Portland VA Medical Center, P3-GI, 3710 SW US Veterans Hospital Road, Portland, OR 97239, USA.

Received: 25 October 2016 Accepted: 30 April 2017

Published online: 05 June 2017

\section{References}

1. Jemal A, Siegel R, Ward E, Hao Y, Xu J, Thun MJ. Cancer statistics, 2009. CA Cancer J Clin. 2009;59(4):225-49.

2. Center MM, Jemal A, Ward E. International trends in colorectal cancer incidence rates. Cancer Epidemiol Biomarkers Prev. 2009;18(6):1688-94.

3. Jones $S$, Chen W-D, Parmigiani G, Diehl F, Beerenwinkel N, Antal T, Traulsen A, Nowak MA, Siegel C, Velculescu VE, et al. Comparative lesion sequencing provides insights into tumor evolution. Proc Natl Acad Sci U S A. 2008; 105(11):4283-8

4. Winawer S, Fletcher R, Rex D, Bond J, Burt R, Ferrucci J, Ganiats T, Levin T, Woolf $\mathrm{S}$, Johnson D, et al. Colorectal cancer screening and surveillance: clinical guidelines and rationale-Update based on new evidence. Gastroenterology. 2003:124(2):544-60.

5. Heitman SJ, Ronksley PE, Hilsden RJ, Manns BJ, Rostom A, Hemmelgarn BR. Prevalence of adenomas and colorectal cancer in average risk individuals: a systematic review and meta-analysis. Clin Gastroenterol Hepatol. 2009;7(12):1272-8.

6. Winawer SJ, Zauber AG, Fletcher RH, Stillman JS, O'Brien MJ, Levin B, Smith RA, Lieberman DA, Burt RW, Levin TR, et al. Guidelines for colonoscopy surveillance after polypectomy: a consensus update by the US multi-society task force on colorectal cancer and the American cancer society. Gastroenterology. 2006;130(6):1872-85.

7. van Ballegooijen M, Rutter CM, Knudsen AB, Zauber AG, Savarino JE, Lansdorp-Vogelaar I, Boer R, Feuer EJ, Habbema JDF, Kuntz KM. Clarifying differences in natural history between models of screening: the case of colorectal cancer. Med Decis Mak. 2011;31(4):540-9.

8. Kuntz KM, Lansdorp-Vogelaar I, Rutter CM, Knudsen AB, van Ballegooijen M, Savarino JE, Feuer EJ, Zauber AG. A systematic comparison of microsimulation models of colorectal cancer: the role of assumptions about adenoma progression. Med Decis Making. 2011;31(4):530-9.

9. Lee GH, Malietzis G, Askari A, Bernardo D, Al-Hassi HO, Clark SK. Is rightsided colon cancer different to left-sided colorectal cancer? - a systematic review. Eur J Surg Oncol. 2015;41(3):300-8.

10. Gupta S, Balasubramanian BA, Fu T, Genta RM, Rockey DC, Lash R. Polyps with advanced neoplasia are smaller in the right than in the left colon: implications for colorectal cancer screening. Clin Gastroenterol Hepatol. 2012;10(12):1395-401. e1392. 
11. Heitman SJ, Hilsden RJ, Au F, Dowden S, Manns BJ. Colorectal cancer screening for average-risk North Americans: an economic evaluation. PLoS Med. 2010;7(11):e1000370.

12. Atkin WS, Edwards R, Kralj-Hans I, Wooldrage K, Hart AR, Northover JMA, Parkin DM, Wardle J, Duffy SW, Cuzick J, et al. Once-only flexible sigmoidoscopy screening in prevention of colorectal cancer: a multicentre randomised controlled trial. Lancet. 2010;375(9726):1624-33.

13. Schoen RE, Pinsky PF, Weissfeld JL, Yokochi LA, Church T, Laiyemo AO, Bresalier R, Andriole GL, Buys SS, Crawford ED, et al. Colorectal-cancer incidence and mortality with screening flexible sigmoidoscopy. N Engl J Med. 2012;366(25):2345-57.

14. Elmunzer BJ, Hayward RA, Schoenfeld PS, Saini SD, Deshpande A, Waljee AK. Effect of flexible sigmoidoscopy-based screening on incidence and mortality of colorectal cancer: a systematic review and meta-analysis of randomized controlled trials. PLoS Med. 2012;9(12):e1001352.

15. Holme $O$, Loberg M, Kalager M, Bretthauer M, Hernan MA, Aas E, Eide TJ, Skovlund E, Schneede J, Tveit KM, et al. Effect of flexible sigmoidoscopy screening on colorectal cancer incidence and mortality: a randomized clinical trial. JAMA. 2014;312(6):606-15.

16. Shroff J, Thosani N, Batra S, Singh H, Guha S. Reduced incidence and mortality from colorectal cancer with flexible-sigmoidoscopy screening: a meta-analysis. World J Gastroenterol. 2014;20(48):18466-76.

17. Bokemeyer B, Bock H, Huppe D, Duffelmeyer M, Rambow A, Tacke W, Koop $H$. Screening colonoscopy for colorectal cancer prevention: results from a German online registry on 269000 cases. Eur J Gastroenterol Hepatol. 2009; 21(6):650-5.

18. Brenner H, Hoffmeister M, Stegmaier C, Brenner G, Altenhofen L, Haug U. Risk of progression of advanced adenomas to colorectal cancer by age and sex: estimates based on 840,149 screening colonoscopies. Gut. 2007;56(11):1585-9.

19. Manser CN, Bachmann LM, Brunner J, Hunold F, Bauerfeind P, Marbet UA. Colonoscopy screening markedly reduces the occurrence of colon carcinomas and carcinoma-related death: a closed cohort study. Gastrointest Endosc. 2012;76(1):110-7.

20. Winawer SJ, Zauber AG, Ho MN, Obrien MJ, Gottlieb LS, Sternberg SS, Waye JD, Schapiro M, Bond JH, Panish JF, et al. Prevention of colorectal - cancer by colonoscopic polypectomy. N Eng J Med. 1993;329(27):1977-81.

21. Quintero E, Castells A, Bujanda L, Cubiella J, Salas D, Lanas A, Andreu M, Carballo F, Morillas JD, Hernandez C, et al. Colonoscopy versus fecal immunochemical testing in colorectal-cancer screening. N Eng J Med. 2012; 366(8):697-706.

22. Burt RW, Cannon JA, David DS, Early DS, Ford JM, Giardiello FM, Halverson AL, Hamilton SR, Hampel H, Ismail MK, et al. Colorectal cancer screening clinical practice guidelines in oncology. J Natl Compr Canc Netw. 2013; 11(12):1538-75.

23. Hewitson P, Glasziou P, Watson E, Towler B, Irwig L. Cochrane systematic review of colorectal cancer screening using the fecal occult blood test (hemoccult): an update. Am J Gastroenterol. 2008;103(6):1541-9.

24. Rutter CM, Savarino JE. An evidence-based microsimulation model for colorectal cancer: validation and application. Cancer Epidemiol Biomarkers Prev. 2010;19(8):1992-2002.

25. Zauber AG, Lansdorp-Vogelaar I, Knudsen AB, Wilschut J, van Ballegooijen M, Kuntz KM. Evaluating test strategies for colorectal cancer screening: a decision analysis for the U.S. Preventive Services Task Force. Ann Intern Med. 2008;149(9):659-69.

26. Wilschut JA, Hol L, Dekker E, Jansen JB, Van Leerdam ME, Lansdorp-Vogelaar I, Kuipers EJ, Habbema JD, Van Ballegooijen M. Cost-effectiveness analysis of a quantitative immunochemical test for colorectal cancer screening. Gastroenterology. 2011;141(5):1648-55. e1641.

27. Lansdorp-Vogelaar I, van Ballegooijen M, Zauber AG, Boer R, Wilschut J, Winawer SJ, Habbema JD. Individualizing colonoscopy screening by sex and race. Gastrointest Endosc. 2009;70(1):96-108. 108 e101-124.

28. Lansdorp-Vogelaar I, van Ballegooijen M, Zauber AG, Boer R, Wilschut J, Habbema JD. At what costs will screening with $C T$ colonography be competitive? a cost-effectiveness approach. Int J Cancer. 2009;124(5):1161-8.

29. Knudsen AB, Zauber AG, Rutter CM, Naber SK, Doria-Rose VP, Pabiniak C Johanson C, Fischer SE, Lansdorp-Vogelaar I, Kuntz KM. Estimation of benefits, burden, and harms of colorectal cancer screening strategies: modeling study for the US preventive services task force. JAMA. 2016; 315(23):2595-609.

30. van Ballegooijen M, Rutter CM, Knudsen AB, Zauber AG, Savarino JE, Lansdorp-Vogelaar I, Boer R, Feuer EJ, Habbema JD, Kuntz KM. Clarifying differences in natural history between models of screening: the case of colorectal cancer. Med Decis Making. 2011;31(4):540-9.

31. Colorectal Cancer Model Profiles [https://cisnet.cancer.gov/colorectal/ profiles.html]

32. Filippakis GM, Zografos G, Pararas N, Lanitis S, Georgiadou D, Filippakis MG. Spontaneous regression of rectal polyps following abdominal colectomy and ileorectal anastomosis for familial adenomatous polyposis, without sulindac treatment: report of four cases. Endoscopy. 2007;39(7):665-8

33. Hoff G, Foerster A, Vatn MH, Sauar J, Larsen S. Epidemiology of polyps in the rectum and colon. Recovery and evaluation of unresected polyps 2 years after detection. Scand J Gastroenterol. 1986;21(7):853-62.

34. Hofstad B, Vatn MH, Andersen SN, Huitfeldt HS, Rognum T, Larsen S, Osnes M. Growth of colorectal polyps: redetection and evaluation of unresected polyps for a period of three years. Gut. 1996:39(3):449-56.

35. Steinbach G, Lynch PM, Phillips RK, Wallace MH, Hawk E, Gordon GB, Wakabayashi N, Saunders B, Shen Y, Fujimura T, et al. The effect of celecoxib, a cyclooxygenase-2 inhibitor, in familial adenomatous polyposis. N Eng J Med. 2000;342(26):1946-52

36. Nishihara R, Wu K, Lochhead P, Morikawa T, Liao X, Qian ZR, Inamura K, Kim SA, Kuchiba A, Yamauchi M, et al. Long-term colorectal-cancer incidence and mortality after lower endoscopy. N Eng J Med. 2013;369(12):1095-105.

37. Schoen RE, Pinsky PF, Weissfeld JL, Yokochi LA, Church T, Laiyemo AO, Bresalier R, Andriole GL, Buys SS, Crawford ED, et al. Colorectal-cancer incidence and mortality with screening flexible sigmoidoscopy. N Eng J Med. 2012;366(25):2345-57.

38. Segnan N, Armaroli P, Bonelli L, Risio M, Sciallero S, Zappa M, Andreoni B, Arrigoni A, Bisanti L, Casella C, et al. Once-only sigmoidoscopy in colorectal cancer screening: follow-up findings of the Italian randomized controlled trial-SCORE. J Natl Cancer Inst. 2011;103(17):1310-22.

39. Brenner $H$, Hoffmeister M, Arndt V, Stegmaier C, Altenhofen L, Haug $U$. Protection from right- and left-sided colorectal neoplasms after colonoscopy: population-based study. J Natl Cancer Inst. 2010;102(2): 89-95.

40. Brenner H, Chang-Claude J, Seiler CM, Rickert A, Hoffmeister M. Protection from colorectal cancer after colonoscopy: a population-based, case-control study. Ann Intern Med. 2011;154(1):22-30.

41. Zheng W, Rutter CM. Estimated mean sojourn time associated with hemoccult SENSA for detection of proximal and distal colorectal cancer. Cancer Epidemiol Biomarkers Prev. 2012;21(10):1722-30.

42. Niv Y, Hazazi R, Levi Z, Fraser G. Screening colonoscopy for colorectal cancer in asymptomatic people: a meta-analysis. Dig Dis Sci. 2008;53(12):3049-54.

43. O'Connell JB, Maggard MA, Ko CY. Colon cancer survival rates with the new American joint committee on cancer sixth edition staging. J Natl Cancer Inst. 2004;96(19):1420-5.

44. Arias E. United States life tables, 2008. Natl Vital Stat Rep. 2012;61(3):1-64.

45. van Rijn JC, Reitsma JB, Stoker J, Bossuyt PM, van Deventer SJ, Dekker E. Polyp miss rate determined by tandem colonoscopy: a systematic review. Am J Gastroenterol. 2006:101(2):343-50.

46. Knudsen AB, Lansdorp-Vogelaar I, Rutter CM, Savarino JE, van Ballegooijen M, Kuntz KM, Zauber AG. Cost-effectiveness of computed tomographic colonography screening for colorectal cancer in the medicare population. J Natl Cancer Inst. 2010;102(16):1238-52.

47. Arora G, Mannalithara A, Singh G, Gerson LB, Triadafilopoulos G. Risk of perforation from a colonoscopy in adults: a large population-based study. Gastrointest Endosc. 2009;69(3):654-64.

48. Gatto NM, Frucht H, Sundararajan V, Jacobson JS, Grann VR, Neugut Al. Risk of perforation after colonoseopy and sigmoidoscopy: a population-based study. J Natl Cancer Inst. 2003;95(3):230-6.

49. Williams AR, Balasooriya BA, Day DW. Polyps and cancer of the large bowel: a necropsy study in Liverpool. Gut. 1982;23(10):835-42.

50. Pendergrass CJ, Edelstein DL, Hylind LM, Phillips BT, lacobuzio-Donahue C, Romans K, Griffin CA, Cruz-Correa M, Tersmette AC, Offerhaus GJ, et al. Occurrence of colorectal adenomas in younger adults: an epidemiologic necropsy study. Clin Gastroenterol Hepatol. 2008;6(9):1011-5.

51. Bokemeyer B, Bock H, Hüppe D, Düffelmeyer M, Rambow A, Tacke W, Koop H. Screening colonoscopy for colorectal cancer prevention: results from a German online registry on 269000 cases. Eur J Gastroenterol Hepatol. 2009; 21(6):650-5.

52. Institute NC: Surveillance, Epidemiology and End Results - SEER 9 Regs Public Use. Available from: www.seercancergov 2004. 
53. Schoepfer A, Marbet UA. Colonoscopic findings of symptomatic patients aged 50 to 80 years suggest that work-up of tumour suspicious symptoms hardly reduces cancer-induced mortality. Swiss Med Wkly. 2005;135(45-46):679-83.

54. Lagarias JC, Reeds JA, Wright MH, Wright PE. Convergence properties of the nelder-mead simplex method in low dimensions. SIAM J Optim. 1998;9(1):112-47.

55. Rex DK, Helbig CC. High yields of small and flat adenomas with highdefinition colonoscopes using either white light or narrow band imaging. Gastroenterology. 2007;133(1):42-7.

56. Cancer trends progress report - 2011/2012 update. National Cancer Institute, NIH, DHHS, Bethesda, MD, 2012.

57. Lam AK, Chan SS, Leung M. Synchronous colorectal cancer: clinical, pathological and molecular implications. World I Gastroenterol. 2014;20(22): 6815-20.

58. Atkin WS, Edwards R, Kralj-Hans I, Wooldrage K, Hart AR, Northover JM, Parkin DM, Wardle J, Duffy SW, Cuzick J, et al. Once-only flexible sigmoidoscopy screening in prevention of colorectal cancer: a multicentre randomised controlled trial. Lancet. 2010;375(9726):1624-33.

59. Bressler B, Paszat LF, Chen Z, Rothwell DM, Vinden C, Rabeneck L. Rates of new or missed colorectal cancers after colonoscopy and their risk factors: a population-based analysis. Gastroenterology. 2007;132(1):96-102.

60. Regula J, Rupinski M, Kraszewska E, Polkowski M, Pachlewski J, Orlowska J, Nowacki MP, Butruk E. Colonoscopy in colorectal-cancer screening for detection of advanced neoplasia. N Engl J Med. 2006;355(18):1863-72.

61. Lieberman DA, Rex DK, Winawer SJ, Giardiello FM, Johnson DA, Levin TR. Guidelines for colonoscopy surveillance after screening and polypectomy: a consensus update by the US multi-society task force on colorectal cancer. Gastroenterology. 2012;143(3):844-57.

62. Kuntz KM, Lansdorp-Vogelaar I, Rutter CM, Knudsen AB, van Ballegooijen M, Savarino JE, Feuer EJ, Zauber AG. A systematic comparison of microsimulation models of colorectal cancer: the role of assumptions about adenoma progression. Med Decis Mak. 2011;31(4):530-9.

63. Zauber AG, Lansdorp-Vogelaar I, Knudsen AB, Wilschut J, van Ballegooijen M, Kuntz KM. Evaluating test strategies for colorectal cancer screening: a decision analysis for the U.S. preventive services task force. Ann Intern Med. 2008;149(9):659-69.

64. Weinstein MC. Principles of cost-effective resource allocation in health care organizations. Int J Technol Assess Health Care. 1990;6(1):93-103.

65. Weinstein MC. Decision rules for incremental cost-effectiveness analysis. Elgar Companion to Health Economics 2006:469-478.

66. Lansdorp-Vogelaar I, Knudsen AB, Brenner H. Cost-effectiveness of colorectal cancer screening. Epidemiol Rev. 2011;33(1):88-100.

67. Sharaf RN, Ladabaum U. Comparative effectiveness and cost-effectiveness of screening colonoscopy vs. sigmoidoscopy and alternative strategies. Am J Gastroenterol. 2013;108(1):120-32.

68. Sonnenberg A, Delco F. Cost-effectiveness of a single colonoscopy in screening for colorectal cancer. Arch Intern Med. 2002;162(2):163-8.

69. Yabroff KR, Lamont EB, Mariotto A, Warren JL, Topor M, Meekins A, Brown ML. Cost of care for elderly cancer patients in the United States. J Nat Cancer Inst. 2008;100(9):630-41.

70. Smitten AL, Simon TA, Hochberg MC, Suissa S. A meta-analysis of the incidence of malignancy in adult patients with rheumatoid arthritis. Arthritis Res Ther. 2008;10(2):R45.

71. Botteri E, lodice S, Bagnardi V, Raimondi S, Lowenfels AB, Maisonneuve P. Smoking and colorectal cancer: a meta-analysis. JAMA. 2008;300(23):2765-78.

72. Willett WC. Diet and cancer - an evolving picture. JAMA. 2005;293(2):233-4.

\section{Submit your next manuscript to BioMed Central and we will help you at every step:}

- We accept pre-submission inquiries

- Our selector tool helps you to find the most relevant journal

- We provide round the clock customer support

- Convenient online submission

- Thorough peer review

- Inclusion in PubMed and all major indexing services

- Maximum visibility for your research

Submit your manuscript at www.biomedcentral.com/submit
Biomed Central 